\begin{tabular}{|c|c|}
\hline 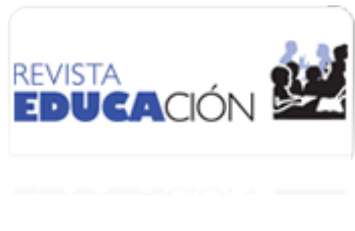 & $\begin{array}{l}\text { Revista Educación } \\
\text { ISSN: 0379-7082 } \\
\text { ISSN: } 2215-2644 \\
\text { revedu@gmail.com } \\
\text { Universidad de Costa Rica } \\
\text { Costa Rica }\end{array}$ \\
\hline
\end{tabular}

\title{
Formación para la tolerancia. Autorregulación de las emociones ${ }^{[1]}$
}

Rodríguez Ortiz, Angélica María; Zapata Blandón, Edilberto

Formación para la tolerancia. Autorregulación de las emociones [1]

Revista Educación, vol. 43, núm. 2, 2019

Universidad de Costa Rica, Costa Rica

Disponible en: http://www.redalyc.org/articulo.oa?id=44058158035

DOI: https://doi.org/10.15517/revedu.v43i2.30068

Esta obra está bajo una Licencia Creative Commons Atribución-NoComercial-SinDerivar 3.0 Internacional. 


\title{
Formación para la tolerancia. Autorregulación de las emociones ${ }^{[1]}$
}

\author{
Angélica Maria Rodríguez Ortiz \\ Universidad Autónoma de Manizales, Colombia \\ angelica.rodriguez276@gmail.com \\ (iD) http://orcid.org/0000-0002-7710-9915 \\ Edilberto Zapata Blandón \\ Universidad Autónoma de Manizales, Colombia \\ zapata.edilber.edilberto@gmail.com \\ http://orcid.org/0000-0001-5006-7279
}

Training in Tolerance - Learning to Self-Regulate Emotions

\author{
DOI: https://doi.org/10.15517/revedu.v43i2.30068 \\ Redalyc: http://www.redalyc.org/articulo.oa?id=44058158035
}

Recepción: 09 Agosto 2017

Aprobación: 17 Junio 2019

\section{ReSUMEN:}

La formación ciudadana ha sido una preocupación central para la filosofía a lo largo del tiempo. Los acontecimientos de las últimas décadas en Colombia muestran el panorama de ausencia de esta y la urgencia de pensar en procesos formativos que la favorezcan. El conflicto en la escuela es solo el reflejo de una sociedad que en esencia es violenta, aun cuando Colombia pasa por un período de postacuerdo; por ello, como docentes se está en la obligación de iniciar al estudiantado en procesos de ciudadanía en los cuales ellos se reconozcan como agentes del cambio social y se involucren de manera consciente en sus procesos. Este artículo presenta los resultados de una investigación en didáctica en el campo de la filosofía, en la cual se trazó como objetivo identificar el papel de la autorregulación de las emociones en la formación para la tolerancia, a partir del reconocimiento del otro como una persona que hace parte del colectivo y con la cual se construye y transforma la realidad social y de espacios de diálogo y argumentación en los que se discutió el problema del bullying. La metodología usada fue la investigación acción, dadas las pretensiones de este proceso, pues formar ciudadanos implica no solo comprender teorías y conceptos, sino ejecutar acciones que den cuenta de ello. El diseño didáctico para la intervención en el aula se efectuó bajo la estrategia de Filosofía para niños y niñas (FpN), para generar espacios de discusión y fortalecer el diálogo y la argumentación.

Palabras Clave: Tolerancia, Autorregulación, Reconocimiento del otro, Convivencia, Filosofía para Niños (FpN).

\section{AbSTRACT:}

Citizenship education has been a significant philosophical concern throughout time. It has also, essentially, been absent from Colombia' for the past few decades. It is adamant to think about educational processes that will favor this aspect. Conflict in the school merely reflects a violent society, even after Colombia's peace agreement that led to a period of peace. Teachers should be obligated to teach their students about citizenship so that they may consider themselves agents of social change and become a conscious part of this transformative effort. This article shows the results of didactic research in the field of philosophy with the objective of identifying the role of self-regulation of emotions when teaching tolerance, based on one's acknowledgement of the other as a part of a collective whole that serves as the basis for society. In this manner, changes in society can occur as well as opportunities for discussion where aspects such as bullying may be addressed. Action research was the methodology used for this study. Educating citizens implies not only understanding theories and concepts, but also, executing actions that display them. The didactic design for intervention in the classroom was conducted according to the Philosophy for Children strategy or enquirebased learning to foster opportunities for discussion.

KEYWORDS: Tolerance, Self-regulation, Acknowledgement of the other, Coexistence, Philosophy for Children (PfC).

\section{INTRODUCCIÓN}

Convivir armónicamente en sociedad exige a los ciudadanos la autorregulación emocional a la hora de orientar sus acciones en el entorno social, así como el reconocimiento del otro como una persona con igual 
condición de derechos. Aprender a convivir con los demás no es una cuestión innata, sino una construcción social en la cual está presente el seguimiento de reglas para demarcar límites entre los intereses propios y el bien común. Las reglas delimitan el actuar humano en la sociedad y a la base de esta debe estar el principio de la tolerancia, como un elemento medular de la construcción social. La diferencia humana exige tener principios y normativas claras a la hora de convivir socialmente; por ello, sin lugar a dudas, ser tolerantes no es una cuestión de elección, sino un fundamento sobre el que opera cualquier normatividad.

Diferencias de pensamiento, de clase, de contexto y otras más, son algo propio a las condiciones humanas y sociales, pero cuando no se controlan estas se convierten en conflictos, dando lugar a que prevalezca la intolerancia. En este sentido, las instituciones educativas no son ajenas a este problema. Por ello, esta propuesta de investigación centró su interés en pensar desde la formación filosófica espacios para reflexionar y discutir el problema del bullying que se presenta en el entorno escolar de la Institución Educativa Santa Ana de los Caballeros, en el Municipio de Cartago, Colombia. Un problema social en el cual se manifiesta la intolerancia de los chicos y chicas en el entorno escolar. Hacer uso de las herramientas que aporta la didáctica de la filosofía, con el fin de fortalecer habilidades para la autorregulación de las emociones, la argumentación (análisis y reflexión) y el diálogo, delimitó el horizonte de esta investigación de aula, con el fin de contribuir en la formación ciudadana basada en el principio de la tolerancia.

El contexto social de las últimas décadas en Colombia ha estado permeado por grandes olas de violencia y las nuevas generaciones no escapan de este devenir histórico. Los niños y las niñas han heredado rasgos violentos y ven en estas formas de actuar ante los problemas la mejor solución. En medio de esta descripción, el municipio de Ansermanuevo Valle del Cauca, no escapa del problema, dado que ha sido una zona afectada por la violencia de grupos armados y de bandas juveniles que se han conformado en los últimos años. Estos rasgos se evidencian en los actuales patrones culturales; pocos ciudadanos de esta zona manifiestan aceptación de las normas legales establecidas para que las personas convivan en el trato del respeto y de la tolerancia por el otro.

Uno de los elementos que acaece a estas situaciones de violencia es el bajo nivel de formación académica en la mayoría de la población; un factor que incide en el desconocimiento de los tratados normativos y el desconocimiento del otro como persona, así como en la participación ciudadana ${ }^{[2]}$. Los habitantes de este municipio no se reconocen como agentes que pueden transformar la realidad social a través de su participación. La influencia cultural del medio social de esta región del país se ve reflejada en el entorno escolar. Las riñas callejeras, el maltrato verbal, el bullying padecido por algunos niños, niñas y jóvenes son, entre otros hechos de intolerancia, emulados en la cotidianidad del entorno educativo de la I.E. Santa Ana de los Caballeros.

Por lo anterior, una vez identificado el problema, se optó por construir en la clase de filosofía un espacio que contribuye en la formación ciudadana. Se elaboró una propuesta, cuya estrategia didáctica retoma algunas herramientas de la propuesta de FpN de Lipman, Sarp y Oscanyan (1992, 2006), por ser este un medio educativo que brinda herramientas didácticas para fortalecer procesos de pensamiento reflexivo, analítico y ético; además, permite a los educandos pensarse como sujetos sociales, como agentes colectivos que crean y transforman intencionalmente la realidad social. La filosofía y la discusión de problemas vivos (bullying) en el aula sirvieron de base para iniciar al estudiantado en un proceso de formación ciudadana en el ámbito escolar basados en el principio de tolerancia.

\section{REVISIÓN TEÓRICA DEL PROBLEMA: ANTECEDENTES Y REFERENTE CONCEPTUAL}

Una vez detectado el problema de la intolerancia en el aula, se inició una revisión teórica para comprender mejor su naturaleza. La necesidad de formar en ciudadanía latente en Colombia se ha convertido en una prioridad, no solo para el cuerpo docente del área de las ciencias sociales, sino para todo el profesorado. Por naturaleza somos seres sociales, además de ser personas que deben aprender a convivir con la diferencia; seres 
humanos que hacen parte de un entorno ciudadano; por ello, todas las áreas del conocimiento deben aportar en la formación ciudadana, de sujetos que piensen críticamente su realidad y la transformen con su saber y actuar. Como lo señala el Ministerio de Educación Nacional (MEN) en Colombia (2011), la obligación es de todo el personal docente de las distintas áreas del saber, puesto que el comportamiento social se convierte en un factor que influye en el rendimiento académico; por ello, ante la grave situación el MEN expone que:

Se ha demostrado que en ambientes escolares donde se presenta menor ocurrencia de vandalismo, violencia física o verbal entre el alumnado, y menor discriminación por razones raciales, lingüísticas, sexuales o sociales, el estudiantado tiende a obtener mejores aprendizajes (Duarte, Bos y Moreno, 2011).

Es por ello que el desarrollo de las competencias ciudadanas y el ejercicio de los derechos humanos se convierten en una condición necesaria para asegurar una educación de calidad, en la medida en que ellas son un camino para la transformación favorable del clima escolar (Duarte et ál., 2011, p. 15).

Así, el problema evidenciado llevó a efectuar una revisión de antecedentes para comprender que en otros países de Latinoamérica, como está ocurriendo en Colombia, se entretejen acciones en las aulas, aportes desde los procesos educativos para formar en ciudadanía.

Serrano (1998), en su trabajo Participación Social y Ciudadana, un Debate del Chile contemporáneo. La ciudadanía activa y las virtudes cívicas, es un ejemplo de ello. La autora manifiesta que en su investigación se evidencia la urgencia de formar para una ciudadanía en todos los espacios. Llega a las siguientes conclusiones en su trabajo de aula:

[...] Se hace necesario: a) un concepto de justicia referido a la inalienable igualdad de los derechos de las personas, asociados a una estructura normativa e institucional que garantiza esas normas de justicia e igualdad de derechos; b) la necesidad de incorporar aspectos culturales y comunitarios en la visión de la ciudadanía y de considerar a quienes son diferentes en el contexto de una creciente sensibilidad multicultural, c) la re elaboración de los conceptos de espacio público y de cultura política y ciudadana, entendidos como los espacios en los que se construye y tiene lugar la ciudadanía (Serrano, 1998, p. 17).

En el mismo sentido, Puig, Domene y Morales (2013) exponen que en los procesos de educación para democracia urgen

resultados pragmáticos y de acción [...] propuestas analizadas pretenden la mejora desde la acción, desde la concienciación y la implicación, de todos los que forman parte de la comunidad educativa. Este uno de los resultados más acordes con el desarrollo de una auténtica Educación para la Ciudadanía (p. 39).

Asimismo, los trabajos de Pérez y Pineda (2016) hablan sobre una formación ciudadana la cual propenda por plantear soluciones para el conflicto que se presenta en la escuela, dado que,

el conflicto en la escuela es producto de las vivencias cotidianas que los niños, las niñas, y los jóvenes experimentan a diario en sus contextos familiares, pero en contravía, a la familia le llegan sus hijos e hijas con conflictos construidos en la escuela y en su paso por la ciudad. Es de anotar que el contexto social que nos envuelve es de violencia generalizada (p. 27).

Aprender a convivir y a tolerar puede ser la base para iniciar a niños, niñas y jóvenes en este proceso.

\section{FORMAR PARA LA TOLERANCIA}

La violencia percibida en el entorno social del Valle del Cauca colombiano y, en particular, de las y los estudiantes de esta zona en su entorno institucional, llevó a pensar cómo desde la escuela se puede enseñar a convivir con los demás y solucionar los conflictos de la mejor manera. Como lo expone Savater (1992)

vivimos en conflicto porque nuestros deseos se parecen demasiado entre sí y por ello colisionan unos contra otros. También es por demasiada sociabilidad (por querer ser todos muy semejantes, por fidelidad excesiva a los de nuestra misma tierra, religión, lengua, color de piel, etc.) por lo que consideramos enemigos a los distintos y proscribimos a los que nos difieren (p. 44). 
Por ello, el conflicto debe tomarse como lo exponen Rodríguez, Andino, Portilla y Portilla (2018) como una oportunidad para convivir con los demás.

Dentro de los referentes conceptuales, además de los planteamientos de Savater (1992), el pensamiento de Voltaire fue central para este proceso, dado que, en su Tratado sobre la tolerancia, en $V$. De cómo la tolerancia puede ser admitida, propone una concepción muy objetiva de tolerancia:

El gran medio de disminuir el número de maniáticos, si quedan, es someter esta enfermedad del espíritu al régimen de la razón que lenta, pero infaliblemente, ilumina a los hombres. Esta razón es dulce, es humana, inspira indulgencia, ahoga la discordia, fortalece la virtud, hace amable la obediencia o las leyes, mucho más de lo que la fuerza las impone (Voltaire, 1978, p. 24).

La tolerancia vista como la facultad humana que valora el reconocimiento del otro abrió un camino y un reto en el proceso formativo, no solo se debe generar estrategias para fortalecer habilidades para la autorregulación de las emociones, sino que además había que llevar al estudiantado al reconocimiento de sí mismo, a partir del reconocimiento del otro. Reconocer las propias conductas, controlarlas y reconocer que hay otros a los que afecto con estas no es una tarea fácil; para ello, se hace necesario aprender a coexistir con los demás en el pleno goce de los deberes, los derechos y las libertades; hecho donde se hace imprescindible la formación de la tolerancia. Ante esta cuestión, Voltaire (1978) expone en VI. De si la intolerancia es de derecho natural y de derecho humano: "No hagas lo que no quisieras que te hagan" (p.26), con lo cual evoca el principio negativo de la regla de oro de la moral; el cual posteriormente retoma Kant en sus imperativos morales. No se comprende, por lo tanto, según tal principio, que un hombre pueda decir a otro: "Cree lo que yo creo y lo que no puedes creer, o perecerás" (Voltaire, 1978, p. 26), sustentando con ello la intolerancia, pues llegaría a ser contradictorio.

La filosofía de Voltaire resulta ser de gran utilidad a la hora de pensar en la realidad violenta padecida por las y las y los estudiantes, pues es común observar los hechos vandálicos, de agresividad, de maltrato, que a diario connotan el incremento del acoso escolar entre los educandos, afectando de esta manera la integridad física, moral y psicológica de las víctimas de este fenómeno en los entornos escolares; lo anterior, dado que elementos como la autorregulación y el reconocimiento del otro se convierten en factores esenciales para que las personas jóvenes tomen conciencia y transformen sus conductas agresivas evidenciadas en sus comportamientos por reflexiones y espacios de convivencia en los que sean tolerantes con sus iguales.

\section{FormaR EN TOLERANCIA DESDE LA AUTORREgULACIÓN DE LAS EMOCIONES}

Para hallar las respuestas objetivas al interrogante planteado en esta investigación fue necesario indagar, además de los autores mencionados otras posturas afines a la formación ciudadana basada en el principio de la tolerancia. Por ello, además del Tratado de la tolerancia; se revisaron los planteamientos de Victoria Camps (2007), quien plantea la necesidad de cultivar la sana convivencia en el marco de la ciudadanía para la tolerancia exponiendo lo siguiente:

Es necesario que las personas se respeten unas a otras y hay que respetar las cosas comunes para que todos las puedan disfrutar cuando las necesiten. El civismo es, por encima de todo, la cultura de la convivencia pacífica y solidaria, del compromiso con la ciudad y con sus habitantes. Queda pendiente saber cómo habrá que formar el carácter de acuerdo a estos valores (Camps, 2007, p. 15).

La formación cívica pone como eje la práctica en relación con el concepto; por ello para comprender lo que significa culturalmente tolerar a los otros es necesario pasar a la acción y realmente tolerarles. La formación ciudadana debe entonces facultar a los individuos para convivir de manera tolerante con los demás y con las normas sociales, es decir, en armonía con las normatividades aceptadas en la interacción social. En otras palabras, la formación en ciudadanía debe propender por el fortalecimiento de habilidades de pensamiento (argumentación) que favorezcan el diálogo como objeto mediador y conciliador de las diferencias; además de habilidades para la autorregulación de las emociones, la cual incluye la toma de conciencia sobre sí mismo a 
partir del reconocimiento del otro. Una convivencia pacífica que dirime la solución dialógica de los conflictos en el ámbito educativo. Para Camps (2007), la convivencia pacífica exige del compromiso de los actores involucrados, los cuales conviven en el marco del respeto.

Por otra parte, se consideraron algunas de las ideas de Silvia Conde (2004) en Rasgos de una Educación para la Democracia, quien expone en torno a la formación ciudadana algunos factores que fueron eje de esta investigación:

[...] En suma, los fines de la educación ciudadana pueden resumirse en la pretensión de formar personas: Con un sólido desarrollo moral, saludables y bien ajustadas a su medio; con capacidad de pensamiento crítico. Personas capaces de construir con otros un orden social que mejore las formas de relación, de funcionamiento social y contribuya a lograr una vida digna para todos. Seres humanos respetuosos de las diversidades y defensoras de la equidad de género, la multiculturalidad y todas las formas de pluralismo. Jóvenes capaces de mejorar las instituciones y procedimientos democráticos, así como de resolver los conflictos de manera no violenta; con un alto sentido de justicia y legalidad, que conozcan la ley y ejerzan sus derechos y deberes fundamentales de las personas y con una visión global y conscientes de las responsabilidades de la humanidad (p. 9).

En la misma línea, Guichot (2012), citando a Raz (1994) y a Salmerón (1998) expone:

La tolerancia es una de esas virtudes esenciales para la convivencia pacífica y el buen hacer de sociedades multiculturales y pluralistas como son las actuales. A ella dedicamos nuestro trabajo, comenzando por dilucidar qué sentido ha de adquirir en nuestros días para responder a los retos del presente, y, más tarde, proponiendo algunas pautas acerca de cómo debería plantearse la formación en esta virtud, en la actitud tolerante, considerándola imprescindible para una auténtica educación emancipadora del individuo (p. 37).

\section{Autorregulación de LAS Emociones. Formación DE HABilidades para LA INTELIGENCIA EMOCIONAL}

Otro elemento esencial en esta investigación fue la autorregulación de las emociones. Por ello, fue menester aproximarse al concepto de autorregulación o autocontrol de las emociones. En este caso fueron fundamentales los aportes de Goleman (1998), quien analiza al sujeto planteando los componentes cognitivos, afectivos, emotivos, de autorregulación emocional, argumentativos, de lenguaje y autocontrol.

\section{AutocontroL}

\section{Mantener bajo control las emociones e impulsos conflictivos}

Las personas dotadas de esta competencia:

- Gobiernan adecuadamente sus sentimientos impulsivos y sus emociones conflictivas.

- Permanecen equilibrados, positivos e imperturbables aun en los momentos más críticos.

- Piensan con claridad y permanecen concentrados a pesar de las presiones (Goleman, 1998, p. 107).

Desarrollar un proceso integral en la formación para la tolerancia demarca como imprescindible trabajar procesos de autorregulación de la mano con el reconocimiento del otro. En este sentido, se inició el abordaje de este concepto con la definición planteada por Brugger (1983): “Tolerancia es el respeto y consideración hacia las opiniones o acciones de los demás" (p. 1594).

Como elementos que subyacen a la autorregulación en este marco conceptual se tornaron esenciales las discusiones que llevaran al estudiantado al reconocimiento del otro. La 'otredad' y 'alteridad' se constituyen en elementos importantes a la hora de formar en ciudadanía, máxime si el principio es la tolerancia, pues reconocer al otro implica reconocerme a mí mismo, a mis acciones e iniciar en procesos de autorregulación para luego pasar a comprender a ese otro, aceptarlo y concebir la existencia de ambos en el mismo mundo social, destinados a construir con los fines mutuos una nueva sociedad. La complejidad social de existir entre 
las diferencias y las necesidades llevan obligatoriamente a la alteridad y la otredad, dado que convocan a ver al otro con sus particularidades, situado en la propia existencia. Desde esta perspectiva, Lévinas (2003) contempla al yo desde el sentido y la relación con el otro desde una mirada ética. En su De otro modo que ser o más allá de la esencia, el autor expone:

Abrirse como el espacio, liberarse mediante la respiración del enclaustramiento en sí mismo supone ya éste más allá, mi responsabilidad para con el otro y mi inspiración por el otro, la carga aplastante —el más allá - de la alteridad. Ciertamente es asombroso que la respiración por la cual los entes parecen afirmarse triunfalmente en su espacio vital sea una consumación, un desnucleamiento de mi substancialidad; que en la respiración me abra ya a la sujeción total al otro invisible; que el más allá o la liberación sea el soporte de una carga aplastante (p. 263).

Con lo cual se delimita el curso de esta investigación, pues, la pretensión era llevar al alumnado a analizar el problema y reconocerse como un ser social, quien son sus acciones afecta al otro, asumiendo aprendizajes que den cuenta del principio de la tolerancia en sus discursos y acciones; exteriorizando el sentido de los comportamientos tolerantes que evidencian autorregulación de las emociones, al dar un buen trato a su compañeros; connotando el respeto, la libertad, la aceptación y el reconocimiento de las partes en común; luego, comprendieron que la tolerancia es la pieza clave para la construcción de la convivencia escolar.

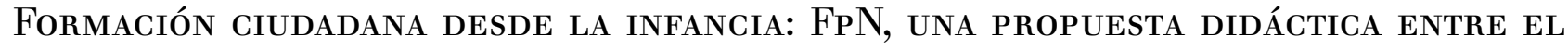 PENSAR, DECIR Y ACTUAR}

Ahora bien, en términos didácticos esta propuesta de investigación se nutrió de los

aportes y herramientas de Filosofía para Niños (FpN), (específicamente, la estrategia didáctica de las novelas de Lisa (Lipman, 1988) y Mark (Lipman, 1989)) — con la cual se potenció en las y los estudiantes su capacidad de reflexión, de análisis y de pensarse como sujetos tolerantes con los otros en el medio escolar - le permitieron al estudiantado asumir las discusiones y argumentaciones razonadas en la perspectiva de la construcción de la convivencia pacífica.

Para llegar a dichos niveles de razonamiento se desarrollaron diversos talleres didácticos que fortalecieron en dichos estudiantes los procesos de la autorregulación y el reconocimiento del otro como virtudes humanas para aprender a tolerar a los demás. De esta forma, $\mathrm{FpN}$ aportó a que los educandos concibieran la tolerancia no solo como un valor, sino también como el fin primordial de la convivencia pacífica en las prácticas escolares; logrando abandonar la intimidación, el maltrato y el acoso como fenómenos de la violencia educativa (acciones que eran opuestas a los principios de la formación ciudadana para la tolerancia).

$\mathrm{FpN}$, aporta herramientas para favorecer procesos de formación en lógica y ética. La propuesta de Lipman, et al. (1992), a partir de los espacios de discusión, el principio del respecto (aunque no se manejó el muñeco de la palabra) sí se actuó bajo este principio; el establecimiento de normas para la discusión, permitió fortalecer procesos de argumentación en el aula. Así, el problema social en el cual estaba inmerso el estudiantado fue el punto de partida para las discusiones y desde los temas de intolerancia abordados en las novelas se fue movilizando el espacio de discusión en los foros y conversatorios.

\section{Metodología}

Dadas las características del problema del bullying presentado en el aula, en el que prima la intolerancia entre el alumnado, se pensó en un diseño metodológico que les vinculara como actores en la solución del problema. En este sentido, se planteó un proceso de investigación de corte cualitativo, con un alcance descriptivo, dado que lo que interesa es interpretar un fenómeno social, intervenirlo y plantear solución desde estrategias didácticas que favorezcan la formación para el ejercicio de la ciudadanía. 
Ahora bien, la formación para la tolerancia a través de la autorregulación de las emociones, así como a través de la argumentación y el diálogo, es algo que no solo se debe trabajar teóricamente, sino que debe involucrar a los sujetos que están inmersos en el problema, para que desde ellos mismos se planteen las soluciones. Visto así, se consideró que la mejor forma para demarcar el camino en esta investigación de aula, era la Investigación Acción (IA); pues permitiría conocer la realidad vivida, producir nuevo conocimiento sobre esta, y pasar a la transformación de dicha realidad. Este método permitió que estudiantes fueran los actores directos de la investigación y con el diseño intencionado de una Unidad Didáctica (UD) en cuya estrategia se usaron algunas de estrategias de FpN y las novelas de Lipman, Lisa (1988) y Mark (1989), se inició el proceso para orientar al estudiantado en pos de la autorregulación de las emociones, así como también en torno a los espacios de discusión que favorecieran la argumentación y el diálogo para aportar en la formación ciudadana, a través del principio de la tolerancia. La IA es un método que permitía, además de conocer lo que ocurre en el aula, llevar a la transformación de la realidad social, por parte de los actores que están inmersos en el problema, ello con el objetivo de alcanzar la sana convivencia en el entorno educativo.

La IA como método de investigación social contempla diversos factores y términos para interpretar la realidad estudiada. Por ello, dadas las pretensiones de formar para la tolerancia se consideró el más pertinente para monitorear en el proceso las acciones y discursos de las y los estudiantes y dar cuenta de los alcances de esta investigación. Además, sus acciones metodológicas permiten una variedad multifuncional de aplicabilidad interdisciplinar para el logro eficaz de las metas propuestas. En términos de Elliot (1993), la IA plantea como objetivo ampliar la comprensión (diagnóstico) de los problemas prácticos del cuerpo docente, proponiendo el "[...] estudio de una situación social con el fin de mejorar la calidad de la acción dentro de la misma” (p. 88); eje neurálgico de la presente investigación.

\section{Unidad de trabajo}

El proceso de investigación se llevó a cabo con 30 estudiantes del grado once (18 mujeres y 12 hombres), cuyas edades oscilaron entre los 15 y 18 años. El diseño de la UD fue intencionado para la asignatura de filosofía, dado que esta estaba siendo reducida a la enseñanza de su historia; y urgía retomar el carácter crítico que esta ofrece, por generar espacios propicios para pensar los problemas esenciales de la sociedad en el marco de la discusión. Además, de brindar herramientas desde la lógica y la ética para argumentar y dialogar de la mano de teóricos que ya han abordado esta serie de problemas a lo largo de la historia de la filosofía.

La intervención didáctica se desarrolló en un período de tres meses, en tres fases continuas. Se efectuaron procesos de monitoreo en el diseño de las actividades que componían la UD, en los talleres, foros y conversatorios diseñados para evidenciar si se presentaban o no avances en los procesos de autorregulación emocional, argumentación y reconocimiento de sí mismo y de los demás como actores en el problema y agentes transformadores.

\section{Instrumentos}

La unidad didáctica para efectuar la intervención en el aula fue diseñada teniendo en cuenta la propuesta de Álvarez (2011), dado que sus lineamientos teóricos facilitó la integración de los ejes temáticos en los procesos de enseñanza y aprendizaje (conceptualización sobre la tolerancia); asimismo, permitió generar espacios de lectura de textos FpN y discusión del problema socialmente vivo del bullying, con el fin de fortalecer la argumentación y el diálogo y llevarles a comprender su papel en la práctica, a partir del análisis de sus conductas y la toma de conciencia sobre la importancia de autorregular sus comportamientos para mejorar sus acciones y tener un buen trato hacia los demás. 
La UD se tomó como un sistema, cuyo diseño consistió en tres fases. Una fase introductoria de la discusión del problema, para indagar por las representaciones sociales que tenía el estudiantado sobre el bullying y el problema de intolerancia en el que estaban inmersos; una segunda fase de discusión y conceptualización, a partir de las lecturas de algunas novelas y cuentos de FpN que representaban el problema social que el alumnado vivía; y una tercera fase de reenfoque, para verificar si había o no progreso en el proceso de formación ciudadana y en el fortalecimiento de las habilidades enunciadas.

Los instrumentos que se usaron para recolectar la información a lo largo del proceso estaban inmersos en la unidad didáctica. Entrevistas semiestructuradas, talleres y foros de discusión fueron esenciales en este proceso. Los talleres y actividades didácticas propuestos desde los acontecimientos presentados en los fragmentos de las novelas Lisa (Lipman, 1988) y Mark (Lipman, 1989) —además de los aportes de autores como Savater (1992, 2004), Aristóteles (2012) entre otros- les permitieron construir diálogos y argumentaciones en torno a la formación de la tolerancia y evidenciar progresos no solo en el discurso, sino en sus comportamientos, ya que la pretensión también apuntaba a la transformación de la realidad y ello solo se lograría en acciones en los que se hiciera evidente la autorregulación de sus emociones.

Los tres momentos de la UD (ubicación, desubicación y reenfoque) fueron desarrollados desde los contenidos filosóficos de $\mathrm{FpN}$, además de vincularon pequeños textos de los aportes epistemológicos de Aristóteles, Voltaire, Savater y Camps, dado que estos pensadores han expuesto el principio de la tolerancia como la virtud más humana que permite a los seres humanos reconocer a los otros como sus iguales y, por ende, conduce a la construcción de escenarios más pacíficos de convivencia (como debería ser la vida escolar).

Así las cosas, la UD tuvo como principio filosófico la propuesta de Lipman, et ál. (1992) en su FpN, dado que:

si queremos ciudadanos adultos que sean racionales con respecto a los valores, deberíamos introducir a los niños en la investigación en valores de tal manera que puedan descubrir por sí mismos que aquello que posee un valor genuino no es el objeto de un deseo cualquiera, en todo caso trivial e inmaduro, sino que más bien es aquello cuya pretensión de ser algo de valor está apoyada por la reflexión y la investigación. (Lipman, et ál. 1992, p. 336).

\section{ANÁLISIS Y DISCUSIÓN DE RESULTADOS}

Las estrategias didácticas que se llevaron a cabo para la formación de la tolerancia y la intervención en el aula con la UD, así como los instrumentos aplicados permitieron recolectar información. Este proceso se grabaron los foros y conversatorios para alcanzar saturación de datos, los cuales se analizaron, y triangularon en cada uno de los tres momentos de la UD.

En este caso, se accedió a la propuesta de la triangulación múltiple expuesta por Denzin (1970); por lo que permitió analizar desde la triangulación de métodos, de actores y de información de acuerdo con las actividades llevadas a cabo en la entrevista semiestructurada, los talleres y los foros. Para aclarar un poco lo abordado en la UD y la recolección de la información, se presenta el Cuadro 1: 


\begin{tabular}{|c|c|}
\hline Momento & Etapas (IP: ideas previas, M: momentos del momento de ubicación). \\
\hline $\begin{array}{l}\text { 1. Ubicación: } \\
\text { Ideas previas. }\end{array}$ & $\begin{array}{l}\text { N. de guias: } 1 \text { y } 2 \text {. } \\
\text { Etapa 1. Indagación IP sobre tolerancia. Etapa 2. Indagación IP sobre reconocimiento del otro. } \\
\text { Etapa 3. Indagación IP sobre autorregulación. Etapa 4. Recolección de información grupal. } \\
\text { Aplicación cuestionarios grupales integrados sobre ideas previas sobre las categorías de la } \\
\text { investigación. }\end{array}$ \\
\hline $\begin{array}{l}\text { 2. Desubicación: } \\
\text { Preconceptuali- } \\
\text { zación. }\end{array}$ & $\begin{array}{l}\text { N. de guias: } 3 \text { y } 4 \\
\text { Etapa 1. Proceso teórico-categorias temáticas. Etapa 2. Indagación, preguntas, respuestas, } \\
\text { asesorias del profesor, videos, caricaturas, historietas y cuentos. Etapa } 3 \text {. Recolección de } \\
\text { información grupal. } \\
\text { Aplicación de encuestas en parejas sobre nuevas conceptualizaciones sobre las categorias de la } \\
\text { investigación. }\end{array}$ \\
\hline $\begin{array}{l}\text { 3. Reenfoque: } \\
\text { Evolución } \\
\text { conceptual. }\end{array}$ & $\begin{array}{l}\text { N. guias: } 5 \text { y } 6 \\
\text { Etapa 1. Profundidad teórica, recapitulación temática: autorregulación, reconocimiento del otro, } \\
\text { ciudadania escolar desde la tolerancia y convivencia pacifica escolar. } \\
\text { Etapa 2. Proceso metacognitivo, reflexión grupal, argumentaciones reflexiones individuales y } \\
\text { grupales; estrategias didácticas: videos, talleres, el aboración de caricaturas, historietas y } \\
\text { dramatizaciones hechas por parte de los estudiantes. } \\
\text { Etapa 3. Desarrollo del pensamiento critico y analitico; evolución conceptual sobre los ejes } \\
\text { temáticos: formación en tolerancia. }\end{array}$ \\
\hline
\end{tabular}

\section{CUADRO 1}

Etapas de la UD Una didáctica filosófica de la tolerancia para la convivencia escolar Fuente: Elaboración propia

Al finalizar la recolección de la información trabajada desde la estrategia didáctica de FpN se ejecutó el análisis de la información aportada por las y los estudiantes en cada uno de los momentos que componían la UD. Este proceso estuvo centrado en analizar e interpretar, las acciones, discursos y argumentaciones, para al final relacionarlos a la luz de las posturas teóricas en concordancia con sus aprendizajes logrados.

\section{FASE 1 EN LA INTERVENCIÓN:}

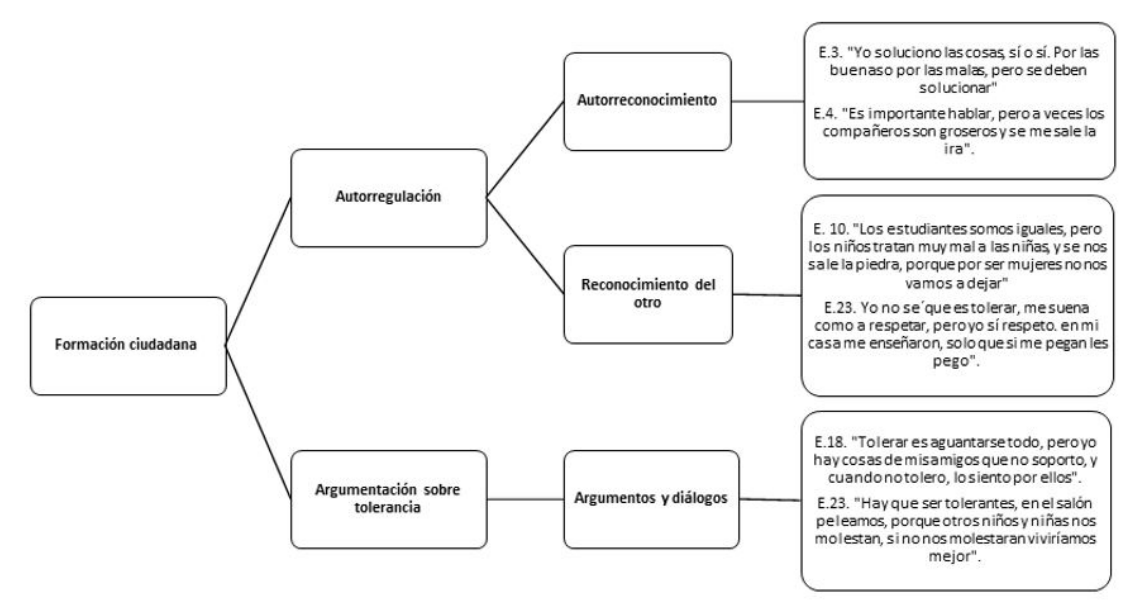

GRÁFICO 1

Momento inicial. Fase 1 de la UD

Fuente: Elaboración propia 
En la primera fase, el discurso de las y los estudiantes, en la entrevista semiestructurada permitió indagar por las representaciones sociales o ideas iniciales que las y los estudiantes tenían sobre el problema de intolerancia en el que estaban inmersos. Desde lo expuesto por autores como Voltaire (1978), Camps (2007) y Santiesteban (2004) la formación ciudadana debe iniciarse desde la infancia y debe hacerse en principios que operen en su realidad escolar, para este caso la tolerancia. Un principio que el estudiantado, al parecer menciona, pero que no se evidencia coherencia de este entre sus discursos y sus acciones. Al inicio de esta intervención, cada estudiante tenía constantemente dificultades entre sí, algunos jóvenes padecían burlas y prácticas de violencia. En el discurso hablaban de tolerancia, pero en realidad no se respetaban ni toleraban las diferencias de género, ni diferencias de ideologías. Además, de caer en contradicciones en sus argumentos, como lo evidencia el discurso del estudiante E.23. en el Gráfico 1 quien manifiesta que ella es tolerante siempre y cuando no la molesten. En otras palabras, enuncian el término, pero no son consistentes con lo que el significado de este convoca, y menos para relacionarlo con sus acciones en el aula.

En el mismo sentido, las primeras intervenciones en la entrevista dejan ver que estos estudiantes son egocentristas, pues no reconocen el valor del otro, solo se reconocen a sí mismos y a sus intereses. No son conscientes de sus acciones y no controlan sus emociones. Desde los planteamientos de Goleman (1998) y Lévinas $(1987,2003)$ se puede inferir que no hay control de las emociones, aún falta maduración de la inteligencia artificial, dado que en sus respuestas manifiestan que si les pegan ellos responden de la misma manera.

En cuanto al reconocimiento del otro, es preciso decir que en esta fase el otro no es reconocido como igual, aunque en uno de los discursos se hable de igualdad, es como si usaran la palabra igualdad desconociendo en significado de esta. El discurso está centrado en la defensa de sí mismos, de sus intereses. Encuentran excusas para justificar sus acciones en contra de los demás.

\section{FASE 2 EN LA INTERVENCIÓN}

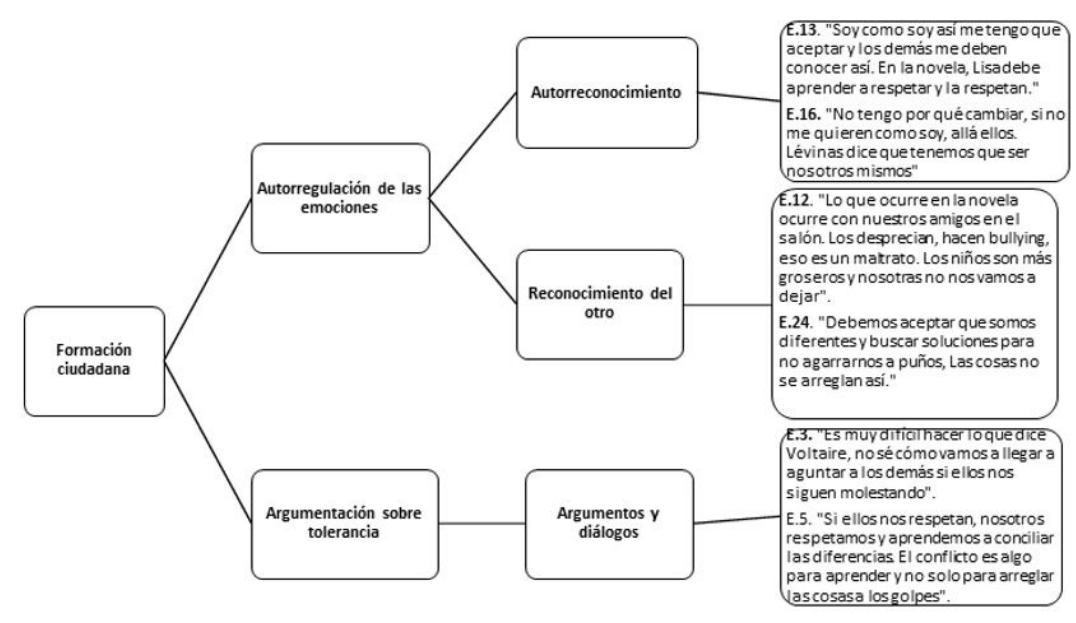

GRÁFICO 2

Momento de desubicación. Fase 2. Apropiación conceptual

Fuente: Elaboración propia

Una vez se dio inicio a la intervención didáctica a partir del diseño de talleres y foros para discutir el problema del bullying, el estudiantado identificó en las lecturas de los textos de FpN de Lipman (1988 y 1989) situaciones similares a las suyas. Situaciones de intolerancia que agreden la convivencia social, como lo expone 
Camps (2007). Las novelas discutidas fueron un pretexto para que los jóvenes se iniciaran en una toma de conciencia, y para que, como lo expone Goleman (1998), se empezaran a evaluar a sí mismos, a reconocerse $\mathrm{y}$ a reconocer sus acciones.

Algunos de ellos, como se muestra en el Gráfico 2, alcanzaron a reconocer que sus acciones afectan a los demás, y que las emociones negativas se pueden controlar. En términos de Rodríguez, et ál. (2018), el conflicto es un espacio para aprender a convivir con otros en la escuela. Estos jóvenes, que están en etapas emocional y psicológicamente de maduración, como lo expone Goleman (1998) deben empezar a trabajar en la introspección, para lograr el autocontrol, al menos se evidencia una pequeña muestra de avance en este aspecto. Asimismo, las acciones efectuadas en las intervenciones en los foros daban cuenta de ellos, pues cuando se interrumpían entre sí, ellos mismos actuaban como moderadores para dar la palabra de manera pausada y con cada docente se enfatizaba en la necesidad de hablar sin gritar y en el marco del respeto por lo que el otro piensa y dice.

En esta fase, se observan que los avances son muy pocos, y ello llevó a reconocer que el ideal de formar para la tolerancia, como lo proponen Camps (2007) o Voltaire (1978) no es una tarea fácil. No obstante, conversatorio a conversatorio, se hacía mayormente evidente el avance tanto en los discursos como en las acciones. Lo anterior se sustenta, dado que las grabaciones evidenciaban que el estudiantado se autorregulaban para participar y controlaban sus emociones cuando alguna de las intervenciones de uno de sus compañeros no les parecía pertinente, ya no se insultaban con palabras soeces y algunos lideraban el proceso de intervención. Asimismo, seguían las reglas de $\mathrm{FpN}$, al respetar el turno en la palabra e interpelar a los demás sin ofenderles.

Es importante enfatizar que en torno a la capacidad argumentativa, en esta fase, no se evidencian muchos cambios en el discurso escrito y oral, pero en la actitud para el diálogo sí, pues recurrían a la palabra y no a los golpes para solucionar sus diferencias. Los discursos siguen siendo cortos, poco sustentados y no superan la fase de la opinión.

En esta fase aún persiste el egocentrismo, no hay evidencia de acciones altruistas y menos de un reconocimiento de la alteridad, pero al menos el hecho de respetar la intervención de los compañeros, esperar para discutir y luego intervenir sin usar términos soeces, se considera un buen avance. Las grabaciones de los diálogos de los foros en esta fase así lo evidencian.

El reconocimiento de sí mismo como agente del cambio es algo que aún no se percibe en una toma de conciencia. En general, podría decirse que, en este aspecto, solo cuatro de los jóvenes se iniciaron en tal proceso, al liderar las discusiones, al recordar a sus compañeros las reglas y al exponer sus propias situaciones vividas en el problema del bullying. Valga aclarar que estas personas eran quienes menos respuestas violentas habían tenido y en general, podría decirse que eran las víctimas del bullying percibido. 


\section{FASE 3 EN LA INTERVENCIÓN}

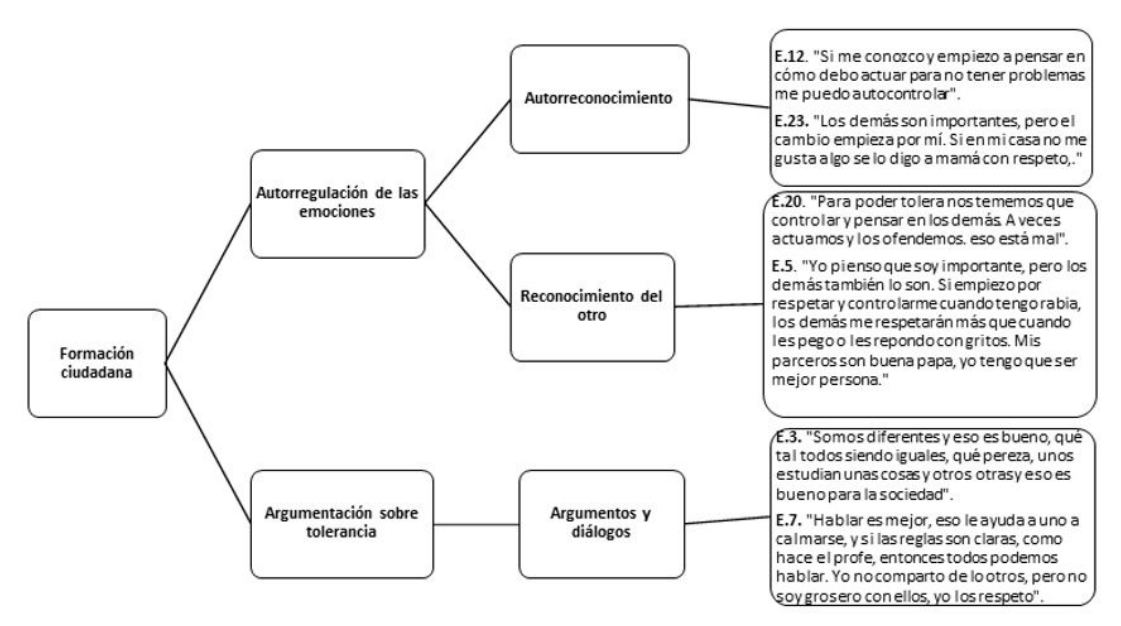

GRÁFICO 3

Momento de reenfoque. Fase 3. Propuestas de reenfoque

Fuente: Elaboración propia

Poco a poco se fueron evidenciando en el proceso avances en el discurso de las y los estudiantes y en las acciones, ante las situaciones de intolerancia presentadas al inicio de la intervención, como se evidencia en el Gráfico 3. Es decir, tres meses atrás. Un progreso que por el corto tiempo puede considerarse como algo importante.

Desde los planteamientos de la tolerancia expuestos por Voltaire (1978) se puede decir que los jóvenes además de discutir sobre sus problemas de bullying a partir de las lecturas también habían avanzado en la comprensión de lo que implica ser tolerantes. Un avance conceptual.

Según sus propios intereses y motivaciones en la discusión orientada por cada docente, y una vez fueron conscientes en el diálogo de las imprecisiones conceptuales en las que estaban incurriendo en los conversatorios, el mismo estudiantado empezó a buscar definiciones sobre el respeto y la tolerancia, así como sobre las cuestiones de lo que implica ser ciudadano. Por ello, también se leyeron algunos apartados del tratado para la tolerancia de Voltaire y de la Política y la Ética para Amador de Fernando Savater (1992, 2004). En sus discursos, poco a poco se fue evidenciando esta apropiación conceptual.

Lo anterior, les llevó a estructurar mejor sus discursos y con ello mejoraron sus procesos de argumentación, como se detalla en la gráfica anterior.

De igual forma, se evidenció que estas discusiones y espacios para el diálogo, así como un juego de roles que se ejecutó en la última fase (propuesto por una de las estudiantes quien sugirió que quienes eran víctimas de bullying actuaran como victimarios y viceversa) les llevó al reconocimiento del otro. Al 'ponerse en los zapatos del otro' reconocieron que no es fácil ser el otro y afectarle con sus acciones. Frases como la del estudiante E.28 "Todos somos iguales y debemos ser tratados como iguales, aunque seamos diferentes en la forma de vivir y pensar. A todos nos rigen las mismas normas" (E. 28, comunicación personal, octubre de 2016) llevaron a inferir avance en este aspecto. Máxime cuando venían de quienes antes fueron víctimas. Este ejercicio les permitió fortalecer ciertas habilidades de argumentación como la reflexión y el análisis de las situaciones, causas y consecuencias, algo que se hizo evidente en el discurso y en el actuar mismo.

Ahora bien, la formación ciudadana es algo que demanda procesos de conocimiento sobre las acciones y la forma cómo estas afectan a los demás. Como lo afirma Camps (2007). Es claro que como docentes se debe formar para la ciudadanía y esa formación debe iniciar con procesos de autocontrol, pero en este aspecto, esta investigación se queda corta, pues el tiempo no es un factor que favorezca. No obstante, aunque fueron 
pocos los avances, es preciso decir, que iniciarles en el conocimiento de sí mismos, llevarles a procesos de introspección para autocontrolarse es un buen inicio que se evidencia en algunos cambios positivos. No se puede decir que todo el estudiantado lo lograron, pero al menos desaparecieron los golpes y las palabras soeces en el aula. Asimismo, como lo expone Lévinas (1987), el reconocimiento de sí mismo inicia al darse cuenta de que hay un otro, uno igual a mí, uno que tiene los mismos derechos que yo tengo. 'Un yo que no soy yo. Un yo que es otro' y en este aspecto también se avanzó de manera gradual.

Ahora bien, la formación para la tolerancia se torna compleja, en la medida en que oscila entre la teoría y la práctica y los espacios diseñados para la discusión. La convivencia social y familiar afectan el proceso, pues son más de quince años en los que estos jóvenes han estado en un entorno en el que se considera 'normal' vulnerar y maltratar al otro. Sin embargo, este no puede ser una limitante y desde la escuela algo se puede hacer. En términos de Walser (1998), con frecuencia se subestima la tolerancia, se toma como si fuera lo menos que se podría hacer por las y los conciudadanos, la mínima expresión de aquello a lo que tienen derecho. Y es que efectivamente la tolerancia, en cuestiones de actitud, toma formas muy diversas. El tolerar (la práctica) puede organizarse de diferentes maneras y la misión como docentes es buscar las mejores maneras para llevar a las y los estudiantes a mejorar sus acciones con los demás.

Cuando se retoma este principio para fundamentar un proceso formativo se reconoce que

la defensa de la tolerancia no es necesariamente una defensa de la diferencia. Puede ser, y con frecuencia no es sino un argumento que brota de la necesidad [...] cada régimen de tolerancia debe ser en cierta medida específico y único, capaz de provocar la lealtad de sus miembros (Walser, 1998, pp. 13, 14).

Esto se ha empezado a evidenciar en el discurso y participaciones de las y los estudiantes, ya que no dan cuenta clara de lo que es la tolerancia, al plantear en sus discursos que tolerar implica aguantar al otro sin involucrarse de manera directa con sus acciones. Quizá esta fue una gran limitante en esta investigación, no solo por la complejidad del significado del concepto 'tolerancia', sino por el ejercicio de esta, dentro de una sociedad en la que se ha privilegiado a la violencia. No obstante, este es apenas el inicio y los resultados del proceso dan muestra de que es posible, además de necesario, seguir trabajando en ello, hasta que las y los estudiantes internalicen que son agentes del cambio social; que sus acciones repercuten en los demás de manera positiva o negativa; razón por la cual, hay que autocontrolar o autorregular, como lo propone Goleman (1998) las emociones y el comportamiento para tener una mejor convivencia con los demás.

Por otra parte, cabe anotar que, al finalizar la intervención en el aula, se logra ver que de 30 estudiantes que hacían parte del grupo de del grado once que se intervino didácticamente, hubo un avance satisfactorio, al menos lo que permite ver un buen inicio de este largo camino. No en todos, pero al menos sí en la generalidad, como se evidencia en el Gráfico 4. 


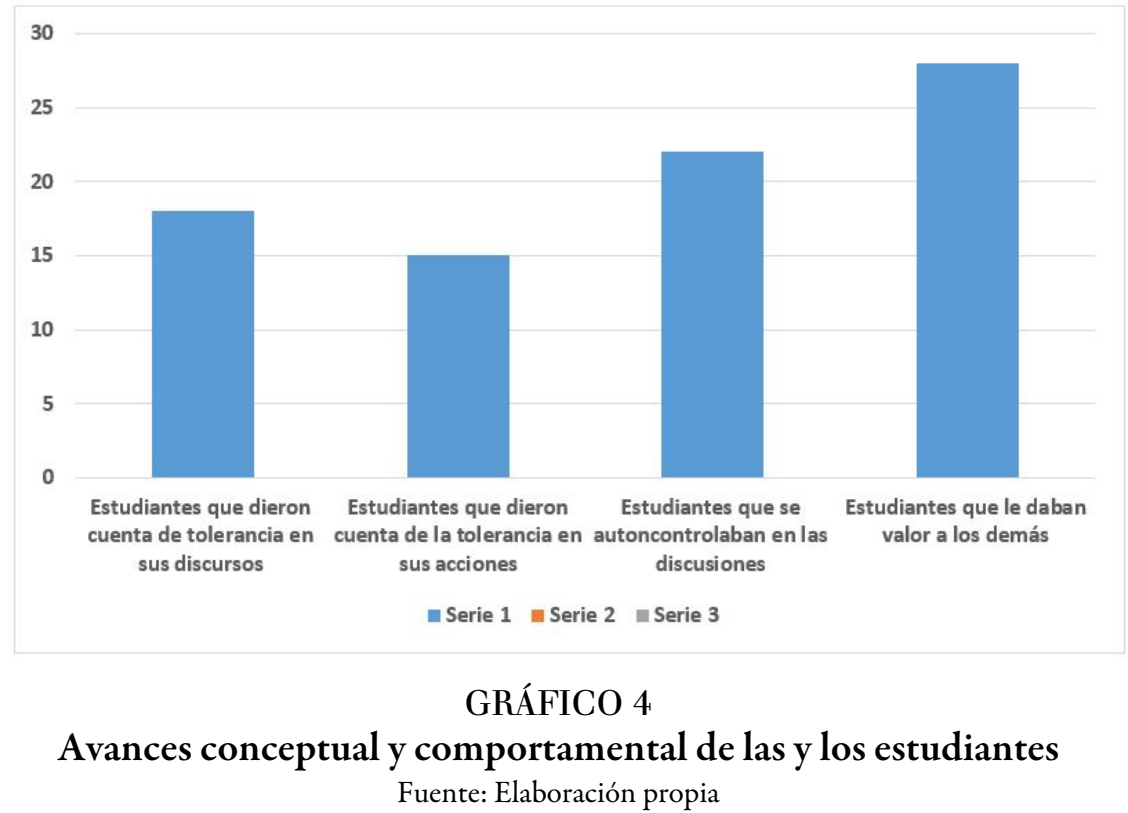

Las evidencias grabadas de las discusiones en los foros y conversatorios; los discursos escritos y las acciones del estudiantado permitieron evidenciar avances que a largo plazo pueden ser relevantes para transformar no solo el contexto escolar, sino el entorno en el cual las y los jóvenes habitan, tal y como se presenta en el Gráfico 4. Aprender a gestionar los conflictos y a movilizar y liderar procesos de discusión se considera que fue uno de los grandes logros del estudiantado; algo que se evidencia en la ausencia de palabras soeces para dirigirse a sus compañeros, así como el hecho de eliminar la solución de conflictos a partir de agresiones personales, estos son quizás los logros más efectivos en este proceso, dado que si bien aún permanecían espacios en los que se evidencia intolerancia con lo que el otro piensa, ya se intentaba hablar en un marco normativo en el que todos son iguales.

\section{CONCLUSIONES}

Al finalizar el proceso de investigación, y partiendo de los resultados obtenidos a partir de la intervención didáctica propuesta, se presentan algunas conclusiones y reflexiones. Entre ellas, se puede afirmar que se materializaron como unidad las situaciones problémicas narradas por el propio alumnado, dado que de este proceso salió material elaborado por cada estudiantes (cartillas y cuentos) para ilustrar estos problemas de intolerancia en el colegio y con ello se trabajará en talleres con estudiantes de otros grados.

El desarrollo de los diferentes ejercicios, talleres y actividades que se ejecutaron en el aula escolar permiten afirmar que sí es posible iniciar procesos para formar en tolerancia, aunque es mejor hacerlo en edad temprana, además de contar con el apoyo de otros agentes como padres y vecinos, para que no haya distancia entre lo que se enseña en la casa y lo que se aprende en el colegio.

La estrategia didáctica al usar el método de $\mathrm{FpN}$ propició espacios de diálogos; espacios de suma importancia para los jóvenes, pues en estos lograron potencializar habilidades para la autorregulación de las emociones, así como habilidades para la argumentación y el planteamiento de otras soluciones diferentes a las que se habían convertido en patrones 'naturales' de su comportamiento. En otras palabras, alcanzaron procesos e introspección que les llevó a iniciarse en la toma de conciencia sobre las acciones propias para autocontrolarse y pasar al reconocimiento del otro, reflexionando del cómo con sus conductas y comportamientos intolerantes afectaban en gran manera la sana convivencia escolar. 
En esta dirección, Lipman $(1988,1989$ y 2006) con su propuesta de FpN aportó elementos relevantes para los educandos desde el trabajo de la Filosofía en el aula, como fueron:

- Mejoramiento de la capacidad de razonar y reflexionar al momento de tratar con los otros.

- Desarrollo de la creatividad y el cuidado a la hora de emitir juicios en situaciones problémicas o de conflictos, anteponiendo el diálogo para solucionarlos pacíficamente.

- Favoreció el crecimiento personal e interpersonal y el trabajo en equipo.

- Potencializaron la sensibilidad ética y el sentido de los otros como sus iguales en la convivencia escolar.

Sus representaciones sociales sobre el conflicto vivido en el aula se tuvieron en cuenta y se inició el trabajo de autorreconocimiento a partir de estas apreciaciones iniciales, para ir monitoreando el proceso y ver si hubo o no avance.

Las discusiones se efectuaron conjuntamente con el profesorado y en cada espacio el establecimiento de normas claras como lo supone $\mathrm{FpN}$ tuvo como principio la tolerancia, a partir de situaciones que presuponían todo lo contrario: la intolerancia. De esta manera, la estructuración de una UD permitió aproximar al estudiantado al problema tanto en lo conceptual como en lo práctico, logrando con ello una toma de conciencia en la que se alcanzó una visión más humana del otro, algo parecido a lo propuesto en el Tratado de la tolerancia en la vida escolar

Asimismo, se logró evidenciar que, al iniciar la fase de las ideas previas, estudiantes desconocían el contexto teórico, comprensivo y de profundidad conceptual que concatenan la autorregulación y el reconocimiento del otro como papeles fundamentales para la formación ciudadana de la tolerancia; y es así como ellos aprendieron que tolerar no significa solo respeto hacia el otro. Lograron concebir que el otro en la escuela trasciende a la esfera del trato propio que se desea recibir; en este caso se acude a Camps (2007), quien propone la tolerancia como la convivencia pacífica que exige del compromiso de los actores involucrados. Dice un estudiante:

Si Todos, los temas, como la autorregulación, y el reconocimiento del otro los aprendimos y sabemos que son papeles muy importantes de la tolerancia y sabemos que los podemos mejorar cada día para avanzar en la solución de la intolerancia escolar de muchos estudiantes habrá que hacerlo (E.8, comunicación personal, octubre de 2016).

Al finalizar la investigación se evidenció que el estudiantado contempló nuevas posturas de sí mismo frente a otro; es decir, aprendieron que la agresividad, la violencia, ni el maltrato hacia los demás son el camino para construir la convivencia pacífica en la interacción escolar.

Es importante anotar que el alumnado presentaba cierta resistencia a los cambios de conductas propuestos por la formación para la tolerancia, pero al implementar la aplicación de la UD toman la iniciativa de darse la oportunidad de aprender de los temas de la tolerancia para, de esta manera, lograr una nueva visión del reconocimiento del otro, de la autorregulación de sus comportamientos y de cómo comprometerse con la construcción de la convivencia pacífica en su entorno escolar. No todos alcanzaron altos niveles en argumentación, y no se puede decir que ya todo lo solucionan con el diálogo, pero el clima escolar ha mejorado, algo bueno, en la medida en que ellos mismos, se reconocen como agentes en épocas de post acuerdo. En términos de uno de las y los estudiantes, "la paz inicia en la escuela, cuando reconocemos a los demás y creemos que con ellos podemos hacer muchas cosas." (E. 30, comunicación personal, octubre de 2016)

Desde esta óptica, el análisis llevó a evidenciar que las y los estudiantes presentaban ideas muy superficiales del concepto de tolerancia y poco sentido de responsabilidad para practicarla en la dinámica escolar; primaban los comportamientos de intolerancia, llegando a la afectación física, psicológica y verbal para con los otros. Luego, el desarrollo de las guías didácticas, los talleres y actividades permitieron que el estudiantado visione hoy una nueva óptica conceptual y práctica del Tratado de la tolerancia desde la comprensión, la reflexión crítica, política, cívica, social, filosófica, antropológica y ciudadana para lograr 
pensarse y comprenderse como el otro, que merece un trato tolerante en el ambiente educativo y fuera de este. Ante esto, los propios estudiantes argumentaron: "Sí tenemos mucha conciencia y una mejor formación en Tolerancia y esto nos hace mejores personas y más Tolerantes con los demás en la INSTITUCIÓN educativa" (E.14, comunicación personal, octubre 2016).

En términos didácticos se podría decir que el grupo reconoció la persistencia de obstáculos, a los cuales debían plantear propósitos organizados para superarlos en el día a día del compromiso escolar con los otros. En el proceso de enseñanza se observó que las conductas, comportamientos, estados emotivos y afectivos que conducían a que estudiantes manifestaran altos niveles de intolerancia, de violencia, de agresividad- se vivenciaban en sus senos familiares; también se logró percibir la influencia del contexto socio cultural de la intolerancia, pues los jóvenes al llegar a las aulas iniciaban conflictos afectando la integridad de los otros y ellos en su discurso lo reconocían. Es el caso de E.13, quien afirma:

A veces somos groseros y agredimos a los demás, porque muchas veces nos tratan mal nuestros padres o en la calle y así sentimos odio o rabias, con todo el que nos mire o nos creemos con mucho poder para querer ser más que los otros y ya sabemos que eso no puede ser así, es mejor controlar los comportamientos y dar mucha tolerancia así, reconocemos el valor y la misma importancia de los otros (E.13, comunicación personal, octubre de 2016).

Es de suma importancia valorar y reconocer el gran compromiso e interés que demostró el estudiantado por los aprendizajes de la formación para la tolerancia y, en esa perspectiva, la investigación logró evidenciar que, la autorregulación y el reconocimiento del otro si cumplen un papel fundamental en la formación de los seres humanos; en este caso, se ha logrado con notable relevancia en el mencionado grupo de estudiantes, la comprensión conceptual y práctica del Tratado de la tolerancia; desde la cual pudieron discernir con criterio responsable y cómo tratar tolerantemente a los otros en el mundo escolar.

Es importante formar en el autoconocimiento y en el respeto por la diferencia, dejar en claro al alumnado que es sujeto ciudadano y que son ellos los actores que pueden transformar la realidad social. Así, el otro se vuelve necesario cuando el deseo propio es el de convivir en sociedady, más aún, en el entorno escolar y con el otro se puede cambiar la realidad social, con palabras y acciones que den cuenta de nuevos ejercicios de ciudadanía.

\section{RefERENCiAS}

Álvarez, O. (2013). Las unidades didácticas en la enseñanza de las Ciencias Naturales, Educación Ambiental y Pensamiento Lógico Matemático. Itinerario educativo, 27 (62), 115-135. Recuperado de:http://revistas.usb.ed u.co/index.php/Itinerario/article/view/1494/1265.

Aristóteles. (2012). Ética a Nicómaco. Madrid, España: Alianza.

Brugger, W. (1983). Diccionario de filosofía. Barcelona: Herder.

Camps, V. (2007). Educar para la ciudadanía. Sevilla, España: Fundación ECOEM.

Conde, S. (2004). Educar para la democracia. La educación ciudadana basada en el desarrollo de competencias cívicas y éticas. México: IFE.

Denzin, N. (1970). Sociological Methods: a Source Book. Chicago, USA: Aldine Publishing Company.

Duarte, J; Bos, M. y Moreno, M. (2011). Los docentes, las escuelas y los aprendizajes escolares en América latina: un estudio regional usando la base de datos del SERCE. Nota Técnica IDB-TN-267. Banco Interamericano de Desarrollo (BID). Recuperado de: http://www.iadb.org

Elliott, J. (1993). El cambio educativo desde la investigación-acción. Madrid: Morata.

Goleman, D. (1998). Inteligencia emocional. Barcelona: Editorial Kairós.

Guichot, V. (2012). Tolerancia, una virtud cívica clave en una educación para la ciudadanía activa, compleja e intercultural. Bordón. Revista de pedagogía, 64 (4), 35-47. Recuperado de https://recyt.fecyt.es//index.php/BO $\mathrm{RDON} /$ article/view/56072/11366.

Lévinas, E. (1987). Totalidad e infinito. Barcelona, Ediciones Sígueme. 
Lévinas, E. (2003). De otro modo que ser o más allá de la esencia. Barcelona, España: Ediciones Sígueme.

Lipman, M. (1988). Lisa. Textos de Filosofia para Niños. Madrid, España: Ediciones De la Torre.

Lipman, M. (1989). Mark. Textos de Filosofía para Niños. Madrid, España: Ediciones De la Torre.

Lipman, M. (2006). Entrevista a Matthew Lipman y a Ann Sharp. Entrevista con Diego Antonio Pineda. Revista internacional Magisterio. 21, 13-16. Recuperado de: https://issuu.com/revistamagisterio/docs/revista_21.

Lipman, M., Sarp, A. M. y Oscanyan, F. (1992). La filosofía en el aula. Madrid: Ediciones de la Torre.

Ministerio de Educación Nacional de Colombia. (2011). Estándares Básicos de Competencias Ciudadanas - Serie Guia No 6: Formar para la ciudadanía si es posible - Lo que necesitamos saber y saber hacer. Bogotá: MEN.

Pérez, I. y Pineda, X. (2016). Gestión educativa aplicada en la integración del aprendizaje activo y la investigación en el área de las matemáticas. Gestión, Competitividad e innovación, 4 (2), 15-26

Puig, M., Domene, S y Morales, J. (2010). Educación para la ciudadanía: referentes europeos. Teoría de la Educación. Revista Interuniversitaria, 22 (2), 85-110.

Rodríguez, A; Andino, M; Portilla, O. y Coral, R. (2018). El conflicto interpersonal como oportunidad para aprender a convivir en la escuela. Análisis, 50 (93), 287-315.

Santiesteban, A. (2004). La formación de la ciudadania: Las TICs y los nuevos problemas. Alicante: AUPDCS.

Savater, F. (1992). Politica para Amador. Barcelona: Ariel.

Savater, F. (2004). Ética para Amador. Barcelona: Ariel.

Serrano, C. (1998). Participación social y Ciudadanía. Un debate del Chile contemporáneo. Chile: Ministerio del Interior.

Voltaire. (1978). Tratado sobre la tolerancia. Madrid: Alfaguara.

Walser, M. (1998). Tratado sobre la tolerancia. Colección Estado y Sociedad. Buenos Aires: Paidós.

\section{Notas}

[1] El presente artículo constituye un apartado de una investigación más amplia presentada en la Maestría en Enseñanza de las Ciencias (MEC) de la Universidad Autónoma de Manizales, Colombia. Sustentada en los grupos de Educación y Cognición y SEAD-UAM de la misma universidad. La investigación fue desarrollada en el municipio de Ansermanuevo, Valle del Cauca, Colombia, en la Institución Educativa Santa Ana de los Caballeros (I. E. SAC).

[2] Prueba de ello son los resultados de las últimas elecciones, las cuales evidencian que un bajo porcentaje de ciudadanos que ejercieron su derecho al voto.

\section{BY-NC-ND}

